\title{
Assessment of Serum Vitamin B12 Levels and Other Metabolic Parameters in Subjects With Different Values of Bone Mineral Density
}

\author{
Marianna Renis ${ }^{\mathrm{a}}$, Debora Lobreglio ${ }^{\mathrm{a}}$, Pierpaolo Congedo ${ }^{\mathrm{b}}$, Maria Concetta Montinaro ${ }^{\mathrm{b}}$, \\ Maurizio Muratore ${ }^{\mathrm{c}}$, Giambattista Lobreglio ${ }^{\mathrm{a}, \mathrm{d}}$
}

\begin{abstract}
Background: Controversial experimental and clinical evidences have raised questions regarding the role of B12 and insulin-like growth factor 1 (IGF-1) on osteoblast function and bone health. In this study, we aimed to determine if the serum levels of B12, IGF-1 and procollagen type $1 \mathrm{~N}$-terminal propeptide (P1NP) are associated with different degrees of bone mineral density (BMD).

Methods: A total of 287 subjects (190 women and 97 men; mean age 53 years) volunteered for evaluation of BMD and serum levels of B12, IGF-1 and P1NP; BMD at lumbar spine and proximal femur was evaluated by means of dual-energy X-ray absorption (DEXA) and expressed as T-score; serum concentrations of vitamin B12 and IGF1 were measured with a chemiluminescent immunoassay on Access II Beckman Coulter and DiaSorin Liaison XL analyzers, respectively; P1NP was assessed in 61 women and 35 men with reduced T-score on Roche Modular platform.
\end{abstract}

Results: A total of 101 subjects (66 women and $35 \mathrm{men}$ ) had a reduced BMD (T-score $<-1$ ) or osteoporosis with a T-score $<-2.5$, while 186 (124 women and 62 men) had a normal BMD. No significant difference in the B12 levels was observed between the subjects with reduced BMD (mean $265.15 \mathrm{pg} / \mathrm{mL}, 95 \% \mathrm{CI}: 236$ - 294.25) and those with normal BMD (mean 243.91, 95\% CI: 225.78 - 262.03) (P $=0.1990)$; lower levels of IGF-1 were observed in the group with reduced BMD (mean $138.7 \mathrm{pg} / \mathrm{mL}, 95 \% \mathrm{CI}: 126.75-150.83$ ) than in that with normal BMD (mean 167.34, 95\% CI: 136.49 - 198.18) $(\mathrm{P}<$ 0.001 ); serum levels of P1NP were significantly lower in 22 subjects younger than 50 years (mean $44.8 \mathrm{ng} / \mathrm{mL}, 95 \% \mathrm{CI}: 36.4$ - 53.1) vs. 74 subjects $>50$ years old (mean 53.3, 95\% CI: $34.3-72.3)(\mathrm{P}<0.001)$, and in women (mean 45.3, 95\% CI: 37.6 - 52.9) vs. men (mean 62, 95\% CI: 23 - 101) $(\mathrm{P}<0.001)$.

Manuscript submitted December 17, 2017, accepted January 3, 2018

aClinical Pathology Unit, "Vito Fazzi” Hospital, ASL Lecce, Italy

'Infectious Disease Unit, "Vito Fazzi" Hospital, ASL Lecce, Italy

"Rheumatology Unit, "Vito Fazzi" Hospital, ASL Lecce, Italy

${ }^{\mathrm{d} C}$ Corresponding Author: Giambattista Lobreglio, Clinical Pathology Laboratory, "Vito Fazzi" Hospital, ASL Lecce, Piazza Muratore Lecce, Italy. Email: patologiaclinica.polecce@ausl.le.it

doi: https://doi.org/10.14740/jocmr3300w
Conclusion: We found no significant association between B12 levels and BMD, but significant associations of lower levels of IGF1 with reduced $\mathrm{BMD}$ and lower levels of $\mathrm{P} 1 \mathrm{NP}$ with younger age and female sex were found; additional studies to further investigate the association of serum levels of B12, growth factors and biochemical turnover markers with human bone health are needed.

Keywords: BMD; Vitamin B12; IGF-1; Calcium; Phosphorus; Parathormone; Vitamin D; P1NP

\section{Introduction}

The bone mineral density (BMD) represents the amount of minerals contained in a bone volume unit $\left(\mathrm{g} / \mathrm{cm}^{3}\right)$ and is conditioned by numerous endocrine-metabolic and nutritional factors, as well as by some genetic polymorphisms, age and lifestyle.

Recent studies indicate that BMD is similar in both sexes before puberty; it is superior in the women at the level of the pelvis and the spine after sexual maturity and becomes greater in humans throughout the skeleton around the $20-22$ years period, reaching the bone mass peak.

After puberty, BMD no longer correlates with age, sex, weight, height, and surface, but reflects instead the increase in the thickness of the trabecular, due in part to the action of latepuberty sex steroids, which modify hormonal and protein and calcium metabolism.

Once the maturity is reached, the skeleton is experiencing a progressive reduction in BMD: for example, women lose $42 \%$ of spinal BMD over the course of life and $58 \%$ of femoral ones.

The skeleton is a complex organ whose development depends on the synergy of bone, cartilage, fibrous and hematopoietic tissue. The particular bone structure, compact and spongy, combines resistance and compactness characteristics ideal for movement. It is also a reserve of calcium, magnesium, phosphorus, sodium and other ions necessary to maintain the homeostasis of many functions of the body. The bone in fact constitutes a huge calcium deposit, which can be mobilized when homeostatic requirements require it.

The physiology of parathyroid hormone (PTH) and calci- 
tonin hormone is closely related to calcium-phosphorus metabolism, with the function of vitamin $\mathrm{D}$ and bone formation.

The mentioned hormones regulate calcium ion concentration, established by intestinal absorption, renal excretion and calcium intake. Calcium homeostasis is closely related to that of phosphorus.

The plasma calcium range is $2-2.6 \mathrm{mmol} / \mathrm{L}$, although only $1 \mathrm{mmol} / \mathrm{L}$ exists as free ionized calcium; only ionized calcium is biologically active, and this is important to most of the functions it carries out [1].

In plasma, calcium is $46 \%$ bound to the circulating proteins and therefore cannot spread through the capillary wall, $8 \%$ is complexed with various ions (phosphate, calcium citrate), and $46 \%$ is present in ionized form [2].

In addition to calcium, other ions are contained in the skeleton. An adult contains about $25 \mathrm{~g}$ of magnesium, twothirds of which are present in the skeleton and one-third in the soft tissues. In bone, magnesium is located on the crystalline surface of hydroxyapatite. Only a small fraction of the bone magnesium can be exchanged with the extracellular. Magnesium is the most important intracellular bivalent cation and is a cofactor for various enzymatic reactions. Finally, an adult contains about $60 \mathrm{~g}$ of phosphorus, of which $80 \%$ is present in the skeleton in crystalline form and $15 \%$ is found in the extracellular liquid in the form of ions, and soft tissues in the form of phosphate esters involved in many biochemical processes [3].

Calcium and phosphorus are both taken up to 1,000 mg/ day. Usually bivalent cations such as calcium ions are poorly absorbed through the intestinal mucosa. In the absence of vitamin D only $10 \%$ or $15 \%$ of dietary and phosphorus calcium is absorbed. The interaction of 1,25(OH)2-D3 with the vitamin D receptor increases the intestinal calcium absorption by $30-40 \%$ and phosphorus by approximately $80 \%$ [4].

The most important factor controlling calcium reabsorption in the nephron distal portions is the PTH, which increases calcium tubular reabsorption and stimulates the kidney to produce 1,25(OH)2-D3 [4].

Among the stimulating factors, bone formation has several growth factors: growth hormone $(\mathrm{GH})$, which likely acts by triggering somatomedin (IGF-1) production; bone morphogenetic protein (BMP), which is contained in the organic matrix of the bone; the bone-derived growth factor (BDGF) that stimulates the synthesis of DNA and bone collagen [5].

The calcium $(\mathrm{Ca})$ and phosphorus $(\mathrm{P})$ ions enter and exit in the bone mineral phase and are under the control of three main hormones: estrogens, parathormone, and metabolites of vitamin D.

Parathormone is a protein hormone synthesized at the level of the main cells of the parathyroid gland, and it is the primary regulator of calcium homeostasis in the blood and bone metabolism. PTH primarily acts with the binding to its PTH1R receptor present at renal and bone levels [6].

Vitamin D plays an important role in the homeostasis of calcium and phosphate and is essential for bone growth and bone integrity. The term "vitamin D" indicates two liposoluble substances: colecalciferol or vitamin D3 and ergocalciferol or vitamin D2. Vitamin D is synthesized through solar exposure and is also taken through diet and supplements.
The active form of vitamin D increases intestinal calcium absorption at the level of the small intestine by interaction with the complex made up of the vitamin receptor and retinoic acid receptor (vitamin D receptor-retinoic acid X-receptor complex (VDR-RXR)), through the increase in the expression of the epithelial calcium channel (transient receptor potential cation channel, subfamily V, member 6 (TRPV6)).

$\mathrm{GH}$, produced by the pituitary gland, acts on the liver, causing the production of growth factors called somatomedin. These stimulate the metabolism and growth of conjugate cartilage chondrocytes, thus promoting bone growth. GH also acts by promoting calcium reabsorption at the renal level, thus contributing to calcium homeostasis.

Procollagen $1 \mathrm{~N}$-terminal propeptide (P1NP) is a bone formation marker, polypeptide residue skinned by the collagen precursor and released into the extracellular fluid, while the central part of the molecule is incorporated into the matrix. Since a single fragment of P1NP is released for each collagen molecule, its measurement provides precise information on the amount of new embedded collagen.

Recent experimental studies on murine models have shown that vitamin B12 plays an important role in bone metabolism by activating the biochemical pathway of growthtaurine-STAT5-IGF-1 which, in turn, is able to control the proliferation, the function of osteoblasts and bone mass. Our study aimed to evaluate any alterations of this pathway in subjects with different BMD values.

During gestation and postnatal period, there is an exponential accumulation of bone tissue that can affect the peak bone mass of adulthood. As a result, both the maternal nutritional state and that of the progeny play a significant role in the genesis of the skeletal apparatus. Recently, the role of taurine and vitamin B12 maternal in the regulation of body growth and BMD of the offspring has been evaluated, as the mechanism by which $\mathrm{GH}$ regulates bone maturation seems to be involved in the synthesis of taurine, a sulphonated amine synthesized in the liver with a STAT5/Vit.B12-dependent mechanism.

Vitamin B12 is a nutrient absorbed in the bowel after binding to the intrinsic gastric factor (GIF) and accumulated in the liver. The deficiency of vitamin B12 and/or taurine has recently been related to the delay in growth and bone maturation. Starting from these assumptions, the study conducted by Roman-Garcia et al [7] has evaluated the role of these two nutrients in a dysfunctional murine experimental model for Gif (KO Gif -/-). In the progeny of homozygous Gif -/- mothers, the absence of vitamin B12 resulted in a clear reduction in body growth and bone mass due to reduced osteoblastic activity. Subcutaneous administration of cyanocobalamin to the mother, in the first gestational phase, or to the offspring from weaning, restored bone growth.

In progeny $\mathrm{KO}$ of heterozygous mothers Gif +/-, however, maternal vitamin B12 levels, albeit reduced, were sufficient at birth to ensure bone growth. In contrast, in the evolutionary age, the progressive consumption of the reserves and the concomitant malabsorption of vitamin $\mathrm{B} 12$ resulted in a reduction in BMD. These KO mice exhibited GH resistance, correlated with reduced IGF-1 tissue phosphorylation, low levels of circulating IGF-1, and decreased hepatic taurine. In addition, 
Table 1. Comparison and Statistical Significance Between the Two Groups of Subjects in the Study

\begin{tabular}{|c|c|c|c|}
\hline & Subject with normal BMD & Subject with reduced BMD & P value \\
\hline Sample dimension (n) & $186(64.8 \%)$ & $\begin{array}{l}101(35.2 \%) \\
\text { osteopenic }(n=75) \\
\text { osteoporotic }(n=26)\end{array}$ & \\
\hline $\begin{array}{l}\text { Average age (years) } \\
\text { Total population }\end{array}$ & $51 \pm 12.54$ & $56 \pm 12.95$ & $\mathrm{P}=0.0003(t$-test $)$ \\
\hline Women & $\begin{array}{l}\mathrm{n}=124(66.6 \%) \\
\text { Average age: } 51 \text { years } \\
95 \% \text { CI: } 49.33-53.36\end{array}$ & $\begin{array}{l}\mathrm{n}=66(65.3 \%) \\
\text { Average age: } 59 \text { years } \\
95 \% \text { CI: } 56.34-61.84\end{array}$ & $\mathrm{P}<0.0001(t$-test $)$ \\
\hline Men & $\begin{array}{l}\mathrm{n}=62(33.3 \%) \\
\text { Average age: } 49 \text { years } \\
95 \% \text { CI: } 45.47-52.76\end{array}$ & $\begin{array}{l}\mathrm{n}=35(34.6 \%) \\
\text { Average age: } 51 \text { years } \\
95 \% \text { CI: } 46.44-56.13\end{array}$ & $\mathrm{P}=0.4724(t$-test $)$ \\
\hline Vitamine B12 (pg/mL) & $\begin{array}{l}\text { Mean: } 243.91 \\
\text { 95\% CI: } 225.78-262.03 \\
\text { SE: } 9.19\end{array}$ & $\begin{array}{l}\text { Mean: } 265.15 \\
95 \% \text { CI: } 236.04-294.25 \\
\text { SE: } 14.67\end{array}$ & $\mathrm{P}=0.1990(t$-test $)$ \\
\hline
\end{tabular}

the reduced STAT5 phosphorylation observed in these $\mathrm{KO}$ animals confirmed the role of vitamin B12/taurine co-operation as a mediator of GH action. Daily dietary taurine supplementation, in these $\mathrm{KO}$ mice lacking vitamin $\mathrm{B} 12$, restored body growth and IGF-1 levels comparable to those of the wild-type strain, while a numerical and functional increase in osteoblasts was evident at bone level.

From these data, it emerges that the above-mentioned effects of taurine are due to increased liver function of IGF-1 and the activation of the receptor for IGF-1 in bone.

The data from this study highlight the role of vitamin B12/ taurine in bone growth and maturation, as possible mediators of the efficiency of the GH/IGF-1 axis. These murine model experimental data open the way for translatory studies in humans about the efficacy of proper dietary supplementation of women of childbearing age to prevent defects in bone growth in the fetus.

\section{Materials and Methods}

A total of 287 volunteer subjects were studied, with an average age of 53 years (range 19 - 85 years), of whom $190(66.2 \%)$ were female and $97(33.8 \%)$ male; they were consecutively subjected to computerized bone mineralometry (MOC) with dual-energy X-ray absorption (DEXA) for the evaluation of BMD at the Rheumatology Outpatient Clinic of the V. Fazzi Hospital; patients with immunoproliferative pathologies and oncological diseases and those receiving phosphonates and/or specific osteoporosis therapy were excluded from the study.

BMD was expressed as standard deviation from the mean bone mass peak (T-score) and was considered normal for Tscore values between -1 and +2.5 , while osteopenia was associated with T-score between -1 and -2.5 , osteoporosis at Tscore $<-2.5$.
The MOC is able to quantify bone mineral content with good approximation and group patients in different risk classes.

"Bone markers" are distinguished by markers for bone formation and bone resorption. The first ones derive essentially from the osteoblast metabolism, the latter being the osteoclast metabolism.

In serum samples, the determination of vitamin B12, vitamin D, GH and PTH was performed using the Access II Beckman Coulter system, with CLIA (immunochemiluminescence) method. The IGF-1 dosage was performed by a single step chemiluminescence sandwich method on the Liaison XL (DiaSorin) analyzer.

The immunoassay for quantitative in vitro determination of total P1NP in serum and human plasma is intended for monitoring the therapy following osteoporosis diagnosis, postmenopausal women and patients with Paget bone disease diagnosis. This immunoassay was performed in electrochemiluminescence (ECLIA) on the Roche analyzer (Cobas modular platform).

Dosing of calcium and phosphorus was performed by colorimetric method using Roche Diagnostics reagents.

\section{Results}

The results in the Table 1 indicate that 186 subjects had normal BMD, while 101 subjects had different degrees of BMD reduction. Unlike the murine experimental model and in accordance with the evidence of literature [8], the serum levels of vitamin B12 in the two groups of subjects were not significantly different, while a statistically significant reduction in IGF-1 levels was observed in the group of subjects with varying degrees of bone density compared to those with normal bone mass; no correlation between IGF-1 and vitamin B12 was observed in 


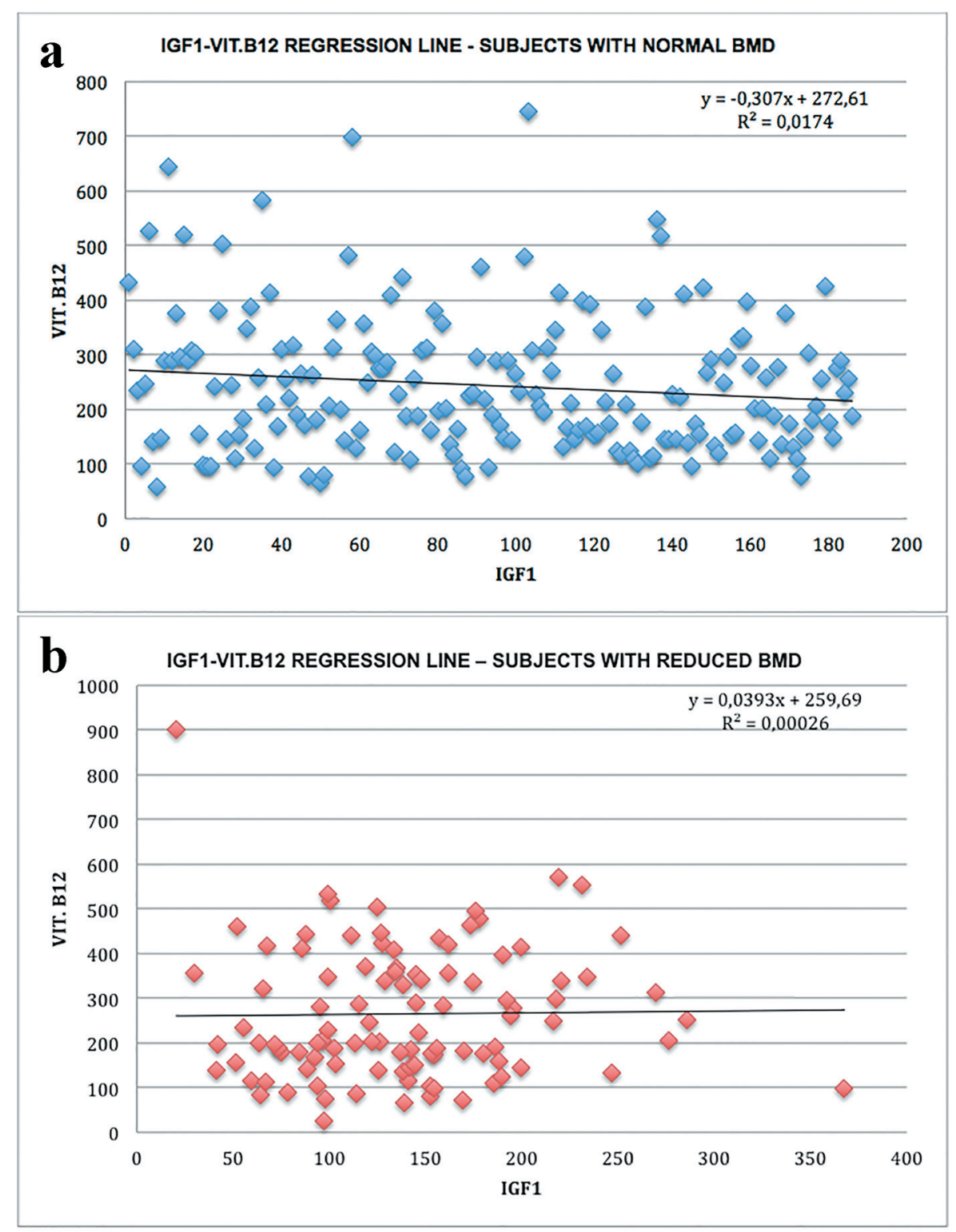

Figure 1. Linear IGF-1-vitamin B12 correlation: regression lines: (a) subjects with normal bone density; (b) subjects with bone mass reduction.

the two groups studied (Figs. 1-3).

Serum levels of P1NP were significantly lower in 22 subjects younger than 50 years (mean $44.8 \mathrm{ng} / \mathrm{mL}, 95 \% \mathrm{CI}: 36.4$ - 53.1) compared to 74 subjects over 50 years (mean $53.3 \mathrm{ng} /$ $\mathrm{mL}, 95 \%$ CI: 34.3 - 72.3) (P<0.001) (Fig. 4); P1NP is lower in women (mean $45.3 \mathrm{ng} / \mathrm{mL}, 95 \%$ CI: 37.6 - 52.9) than men (mean 62 ng/mL, 95\% CI: 23 - 101) (P<0.001) (Fig. 5).

\section{Discussion}

The results obtained confirm that the reduction of IGF-1 levels is associated with significant bone mass reduction, which is an important risk factor for bone fractures; the absence of differ- ence between the serum B12 levels between the two groups of subjects may depend on the fact that total vitamin levels were measured rather than biologically active levels. Further studies are therefore needed to define the role played by the bioavailability of vitamin B12 in determining the development and maintenance of bone mass.

Lower levels of P1NP were found with younger age and female sex. A limitation is because the P1NP is not specific to the bone, although this is the far more important source because of the rapidity of its turnover. Despite the availability of highperformance methods, its use in the clinic is for the time being limited to the evaluation of the response to anabolic drugs [9].

In the bone $1,25(\mathrm{OH}) 2-\mathrm{D} 3$ cause an increase in resorption, depending on calcium levels; this action is synergistic with 


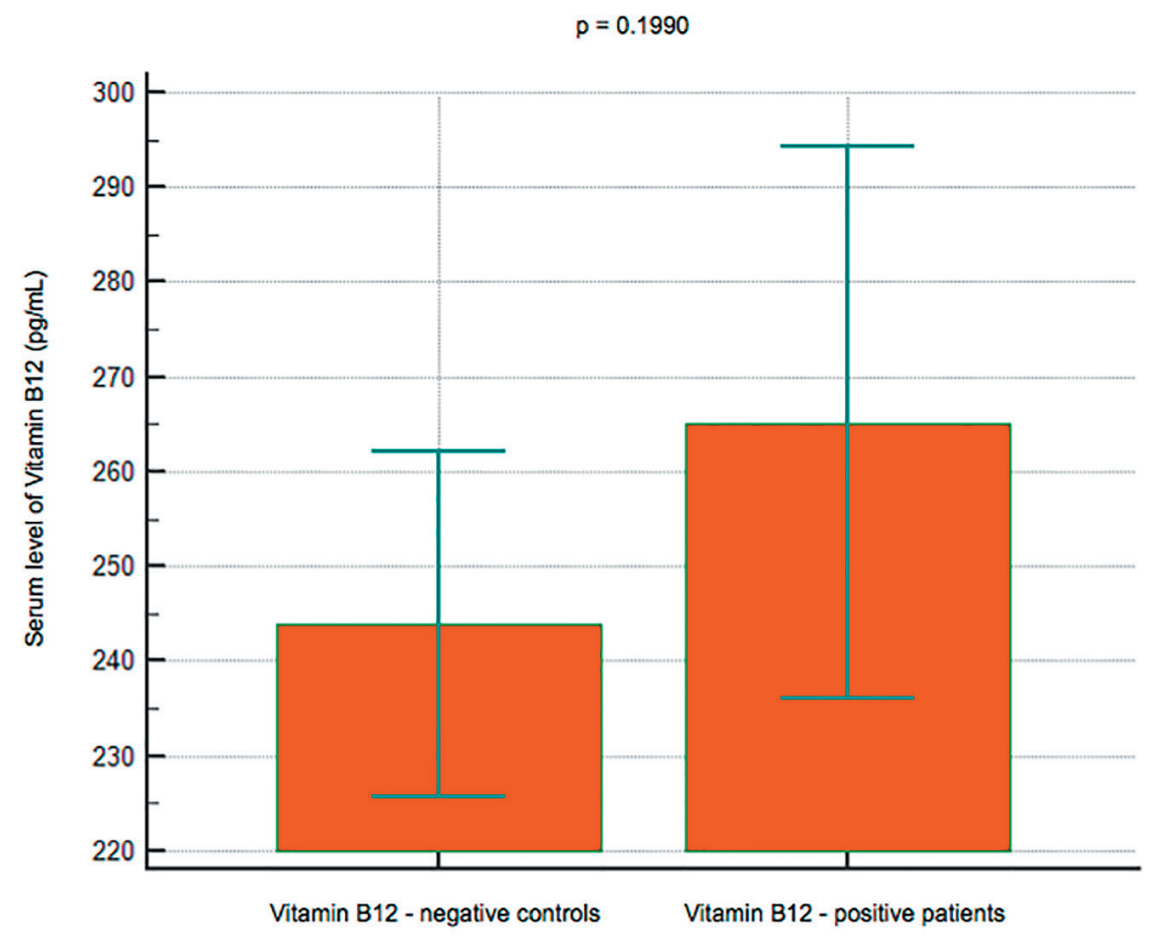

Figure 2. Data comparison graph between negative and positive controls in vitamin B12 measurement.

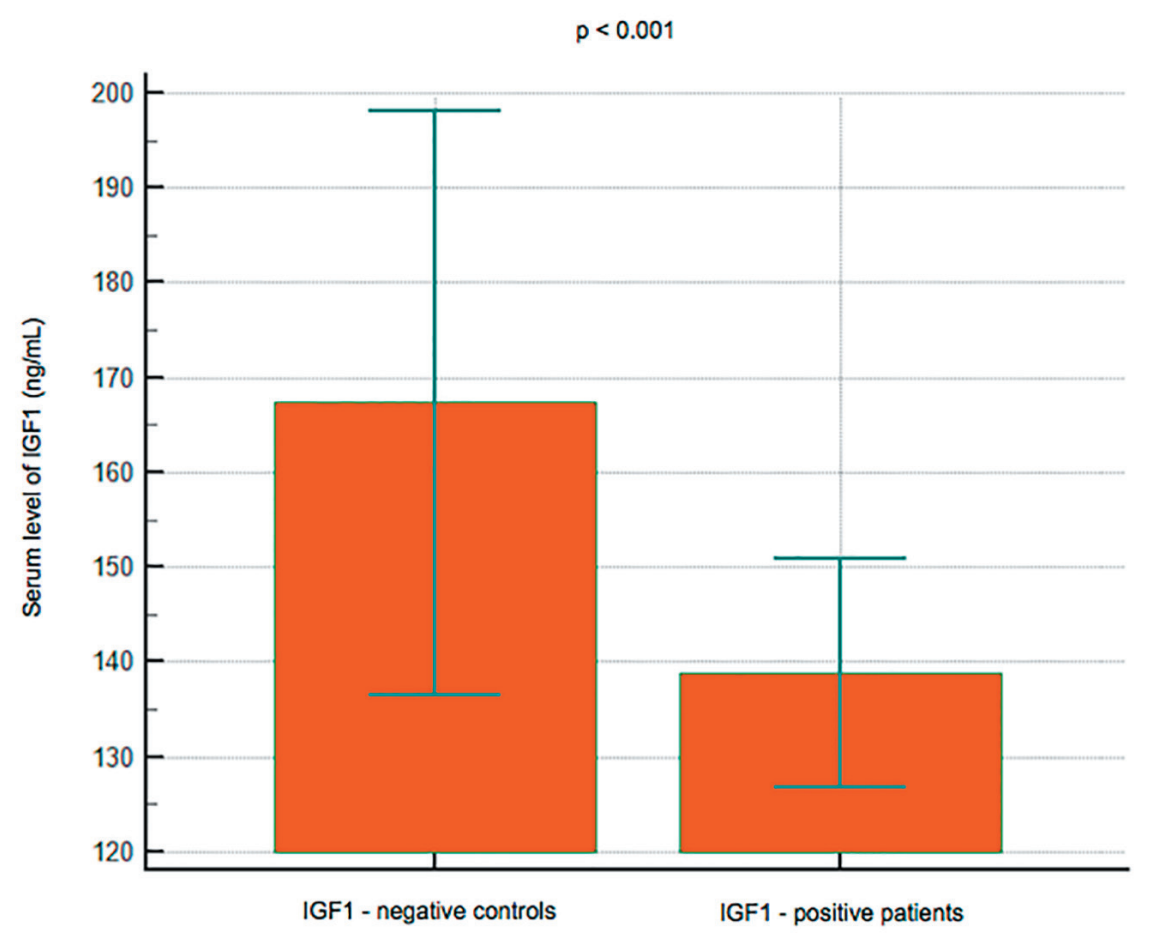

Figure 3. Data comparison graph between negative and positive controls in IGF-1 measurement. 


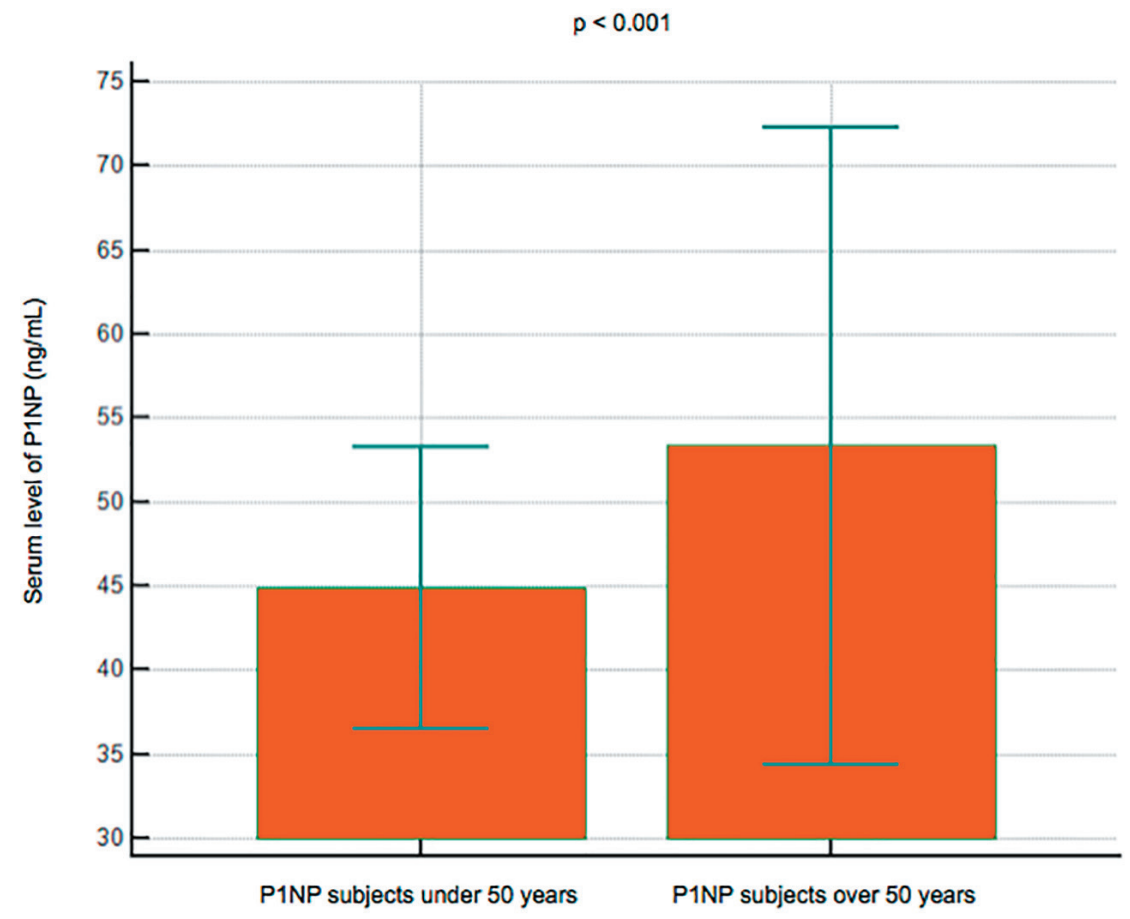

Figure 4. Data comparison graph between P1NP serum levels in subjects under and over 50 years.

that of PTH. 1,25(OH)2-D3 is recognized by the receptors present on the osteoblasts, causing an increase in the expression of the receptor activator of nuclear factor-kB ligand (RANKL) receptor. RANKL binds to RANK, a receptor present on preosteoclasts, stimulating their maturation and thus to become mature osteoclasts, which remove calcium and phosphorus

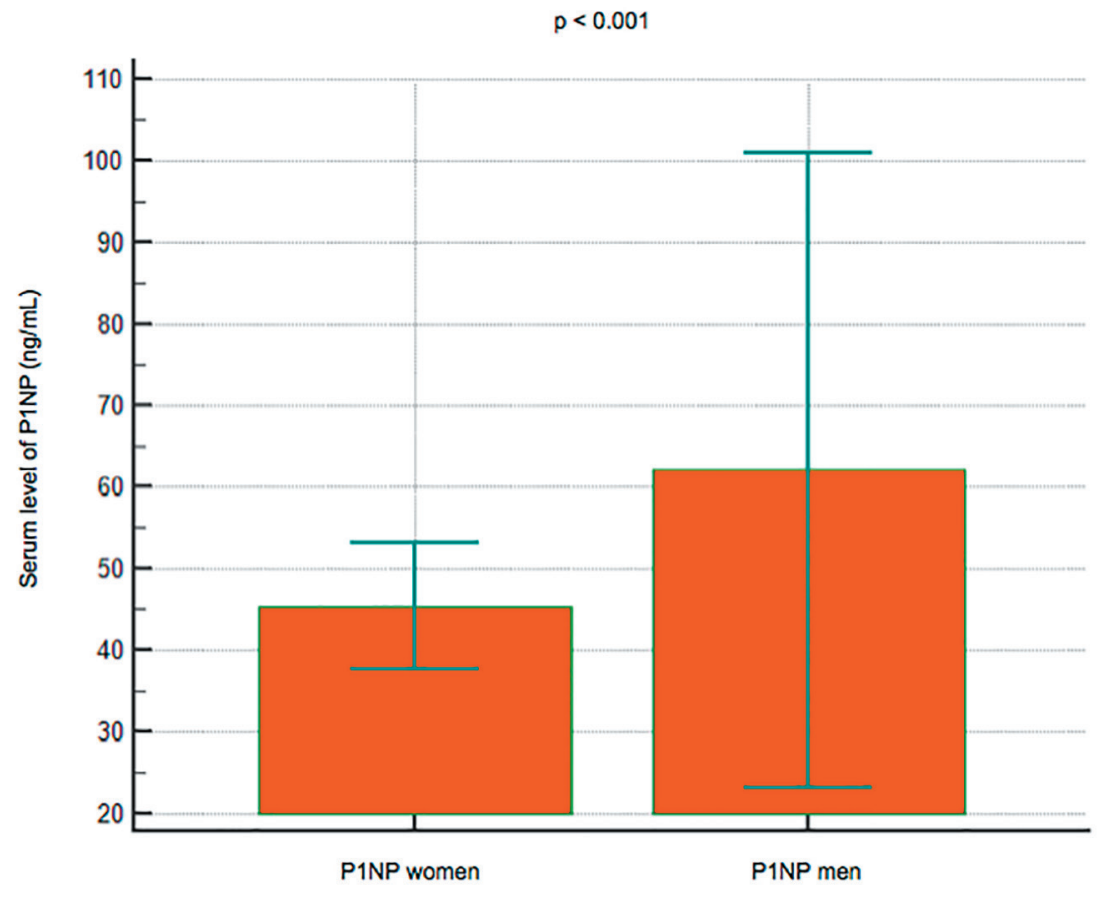

Figure 5. Data comparison graph between P1NP serum levels in women and men. 
from the bone, maintaining adequate plasma and phosphorous levels that promote the mineralization of the skeleton.

Directly or indirectly, 1,25(OH)2-D3 controls more than 200 genes, responsible for cell proliferation regulation, cell differentiation, apoptosis and angiogenesis.

The vitamin D receptor gene (VDR) was related to BMD and some studies have suggested that it may be involved in determinism of osteoporosis [10]. In fact, a VDR polymorphism was identified in part $3^{\prime}$ of the gene contained in chromosome 12 and two alleles named B or b were identified on the basis respectively of the absence or presence of the BSM-I endonuclease restriction site. The BB genotype appears to be associated with significantly lower BMD levels than the bb genotype and is currently studying correlation with other densitometric or biohumoral parameters of bone metabolism [11].

In conclusion, our study did not find a significant association between B12 serum level and BMD; however, additional studies are needed to further investigate the association of this vitamin and other biochemical markers with different degrees of BMD.

\section{Acknowledgments}

All the listed authors have actively participated in groundwork, research and tuition of this current work. We thanks Beckman Coulter Italy, DiaSorin and Roche for kindly providing reagents for the evaluation of metabolic parameters.

\section{Conflict of Interest}

The authors do not have any conflict of interest with regard to the studies described in this manuscript.

\section{References}

1. Nussey SS, Whitehead SA. Principles of Endocrinology,
Chapter 1. Endocrinology An Integrated Approach. 2001.

2. Guyton Arthur C, Hall John E. Parathyroid hormone, calcitonin, calcium and phosphate metabolism. Vitamin D. Bone and teeth; Chapter 79. Medical Physiology. 2006.

3. Broadus AE. Physiologic functions of calcium, magnesium and phosphorus. Primer on the Metabolic Bone Disease and Disorders of Mineral Metabolism, Favus MJ. (ed), Kelseyville, California. 1990;7:29-30.

4. Holick MF. Vitamin D deficiency. N Engl J Med. 2007;357(3):266-281.

5. Passeri G, Girasole G, Manolagas SC, Jilka RL. Endogenous production of tumor necrosis factor by primary cultures of murine calvarial cells: influence on IL-6 production and osteoclast development. Bone Miner. 1994;24(2):109-126.

6. Muller M, Gagiannis S, Nawroth PP, Brune M, Schilling T. Activation of the receptor for parathyroid hormone and parathyroid hormone related protein induces apoptosis via the extrinsic and intrinsic signaling pathway. Int J Mol Med. 2009;24(3):373-380.

7. Roman-Garcia P, Quiros-Gonzalez I, Mottram L, Lieben L, Sharan K, Wangwiwatsin A, Tubio J, et al. Vitamin $\mathrm{B}(1)(2)$-dependent taurine synthesis regulates growth and bone mass. J Clin Invest. 2014;124(7):2988-3002.

8. Cagnacci A, Bagni B, Zini A, Cannoletta M, Generali M, Volpe A. Relation of folates, vitamin B12 and homocysteine to vertebral bone mineral density change in postmenopausal women. A five-year longitudinal evaluation. Bone. 2008;42(2):314-320.

9. Albanese CV, Passariello R. Osteoporosis and bone metabolic diseases - Clinical and Diagnostic. Part IV, Chapter 25: Laboratory Diagnostics. Pages 276-277.

10. Carling T, Kindmark A, Hellman P, Lundgren E, Ljunghall S, Rastad J, Akerstrom G, et al. Vitamin D receptor genotypes in primary hyperparathyroidism. Nat Med. 1995;1(12):1309-1311.

11. Brown EM. Physiology and pathophysiology of the extracellular calcium-sensing receptor. Am J Med. 1999;106(2):238-253. 